\title{
Hyperfrontality and hypoconnectivity during refreshing in schizophrenia
}

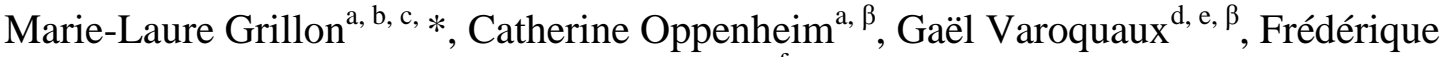 \\ Charbonneau $^{\mathrm{a}}$, Anne-Dominique Devauchelle ${ }^{\mathrm{f}, \mathrm{g}}$, Marie-Odile Krebs ${ }^{\mathrm{a}}$, Franck Bayle ${ }^{\mathrm{a}}$, \\ Bertrand Thirion $^{\mathrm{d}}$, and Caroline Huron ${ }^{\mathrm{f}, \mathrm{g}}$.
}

${ }^{\beta}$ : Catherine Oppenheim and Gaël Varoquaux equally contributed to this work.

${ }^{a}$ : Inserm U894, Université Paris Descartes, Centre de $\quad$ d : Parietal, INRIA, Gif/Yvette, France

Psychiatrie et Neurosciences, Centre Hospitalier Sainte- e : LNAO, DSV/I2BM, NeuroSpin Center, Gif/Yvette, Anne, Paris, France France

${ }^{\mathrm{b}}$ : Laboratoire Mémoire et Cognition, Institut de Psychologie, Université Paris Descartes, BoulogneBillancourt, France

${ }^{c}$ : Service de Psychiatrie Générale, Département de ${ }^{\mathrm{f}}$ : Cognitive Neuroimaging Unit, Inserm U992, CEA, NeuroSpin, Gif/Yvette, France

g : Collège de France, Paris, France Psychiatrie, Centre Hospitalier Universitaire Vaudois (CHUV), Lausanne, Suisse

\footnotetext{
* Address reprint requests to Marie-Laure Grillon, Ph.D. Present address: Laboratoire Mémoire et Cognition, Institut de psychologie, Université Paris Descartes, 71 avenue Edouard Vaillant, 92774 Boulogne Billancourt, France ; +33 (0)1 55205958 ; mlgrillon@gmail.com.
}

\section{Abstract}

Anomalous activations of the prefrontal cortex (PFC) and posterior cerebral areas have been reported in previous studies of working memory in schizophrenia. Several interpretations have been reported: e.g., neural inefficiency, the use of different strategies and differences in the functional organization of the cerebral cortex. To better understand these abnormal activations, we investigated the cerebral bases of a working memory component process, namely refreshing (i.e. thinking briefly of a justactivated representation). Fifteen patients with schizophrenia and 15 control subjects participated in this fMRI study. Participants were told that whenever they saw a word on the screen, they had to read it silently to themselves (read and repeat conditions), and when they saw a dot, they had to think of the just-previous word (refresh condition). The refresh condition (in comparison with the read condition) was associated with increased activation in the left inferior frontal gyrus $(T=3.55, p=0.009)$ and decreased connectivity within the prefrontal cortex and between the prefrontal and parietal cortices $(T s>4.09, p s<0.05)$ in patients with schizophrenia in comparison with control subjects. These results suggest that prefrontal dysfunctions in schizophrenia might be related to a defective ability to initiate (rather than to execute) specific cognitive processes.

Keywords: schizophrenia, executive functions, working memory, prefrontal, refreshing, connectivity 


\section{Introduction}

Numerous neuroimaging studies have reported dysfunction of the lateral (most frequently the dorsolateral) prefontal cortex (PFC) in schizophrenia. The dominant explanation is based on findings that both hypo-activations and hyper-activations are found in patients with schizophrenia depending on the working memory load. These studies have used the term inefficiency to describe this pattern of results, as they note that schizophrenia patients must devote more cognitive resources (or more blood oxygen level dependent response) than control participants to perform the same task (Callicott et al., 2003; Manoach, 2003; Potkin et al., 2009; Kim et al., 2010). Specifically, it has been proposed that the PFC response to increasing memory load in healthy people and people with schizophrenia conforms to an inverted U-shaped curve, whereby neural processing (and fMRI signal) in the PFC increases as working memory load increases, and decreases as working memory capacity is exceeded. Patients with schizophrenia, compared to healthy subjects, would show a leftward shift in the U-shaped curve leading to PFC hyper-activations when performing lowload working memory tasks and PFC hypo-activations when performing high-load working memory tasks in comparison with control subjects. According to this account, schizophrenia patients appear hyper-frontal and hypo-frontal relative to controls at low and high working memory loads, respectively.

However, abnormal activations have been observed in variable PFC areas and in other more posterior (temporal and parietal) cerebral regions. The nature (hypo- or hyperactivation) and localization of these anomalies vary according to the cognitive load, the engaged processes (e.g., maintenance or manipulation), the step of processing (encoding, delay or recuperation phases) or patients performances (impaired or not). These data highlight the complexity of the relationship between cerebral anomalies and cognitive impairments of patients with schizophrenia (Lewis and Anderson, 1995; Minzenberg et al., 2009). Moreover functional integration anomalies have been early assumed to be at the core of schizophrenia (Andreason et al., 1999; Friston, 2005). A number of functional neuroimaging studies have shown aberrant coordination among distant cortical areas in schizophrenia during cognitive tasks (for reviews, see Pettersson-Yeo et al., 2011; Fornito et al., 2012). These lead to the argument that lateral PFC dysfunction must be assessed within the function of larger cortical networks.

When schizophrenia-related abnormal activations are observed in areas that healthy controls commonly use for the task, the abnormal activations are generally interpreted as inefficiency. However, anomalies are not always, and even rarely, seen in areas commonly used for the task. In these cases, several explanations might be suggested. They might reflect a compensatory mechanism, i.e. the use of different strategies (a combination of different processes) from those of control subjects and/or supplementary processes (Quintana et al., 2003). A commonly observed compensatory mechanism in schizophrenia is the overrecruitment of the ventrolateral PFC during dorsolateral PFC-taxing tasks (Tan et al., 2005, 2006; Minzenberg et al., 2009; see also Kim et al., 2010). In addition to ventrolateral PFC hyper-activation, Tan et al., (2006) have showed that ventrolateral PFC activation correlated with accuracy in patients with schizophrenia while dorsolateral PFC activation correlated with accuracy only in control subjects. They suggested that the dorsolateral PFC inefficiency in schizophrenia resulted in compensatory activation from the ventrolateral PFC (i.e., an overuse of maintenance rehearsal).

Alternatively, abnormal activations might be due to a lower functional segregation, i.e., a less well circumscribed functional specialization of given brain regions. For example, it has been suggested that the over-recruitment of the ventrolateral PFC during dorsolateral 
PFC-taxing tasks could be linked to a lower dorsolateral PFC and ventrolateral PFC specialization in working memory manipulation and maintenance respectively (Tan et al., 2005, 2006). More recently, Woodward et al., (2011) showed reduced segregation between the default mode and executive control networks in the prefrontal cortex of patients with schizophrenia. In control subjects, there was very little spatial overlap between the default mode network and other networks and even adjacent cortical regions showed markedly different connectivity patterns. However, in contrast to control subjects, the left inferior frontal gyrus and the left middle frontal gyrus in patients with schizophrenia did not show greater connectivity with either the default mode network or the executive control network. These findings suggested that the functional specialization of prefrontal cortical regions was altered in schizophrenia.

The tasks typically used in working memory studies (e.g., the n-back task) are complex and can be resolved using several different strategies (Conway et al., 2007; Camos et al., 2011). Using these complex tasks, we cannot exclude that abnormal activations in patients with schizophrenia reflect the use of different strategies (a combination of different processes) from those of control subjects and/or supplementary processes. To better understand these abnormal activations, it is thus critical to more specifically relate working memory component processes to patterns of cerebral activity in schizophrenia. In the present study, we sought to investigate cerebral bases of an elementary working memory process, refreshing, in patients with schizophrenia. Refreshing consists in briefly thinking of a just-activated representation of a thought or percept (Johnson and Hirst, 1993; Johnson et al., 2005). The result of refreshing is to increase and/or prolong activation of information that would otherwise quickly become less available. It serves to foreground a representation with respect to other representations that are currently active, thus making the item a focus of reflective attention (Sperling, 1960; Raye et al., 2007; Chun and Johnson, 2011). It helps keep information active in working memory (Camos et al., 2011) and encoding information in episodic memory (Grillon et al., 2008). Johnson and colleagues (Johnson et al., 2002) have developed an experimental procedure to investigate the refresh process. Participants read aloud unrelated words presented one after another on a computer screen. Critical words are presented once (read condition), immediately repeated (repeat condition), or followed by a dot signaling the participants to think of the just previous word and to say it again (refresh condition). The use of this procedure in neuroimaging studies highlight activities in the lateral PFC and the inferior parietal lobule/supramarginal gyrus (Raye et al., 2002; Johnson et al., 2005). Interestingly, a PFC transcranial magnetic stimulation (TMS) deactivation causes a selective slowing of response times to refresh words while a TMS deactivation of the inferior parietal lobule causes a slowing in both refreshing and repeating words (Miller et al., 2008). This suggests top-down signals from the PFC to the inferior parietal lobule underlie response times to refresh. Consistent with this study, Johnson and colleagues show that the disproportionate slowing in response times to refresh in older healthy adults is associated with a single decreased activity in the left dorsolateral PFC (Johnson et al., 2004). In addition, in contrast to healthy young adults, older adults do not benefit from refreshing at encoding to increase long term memory (Johnson et al., 2002). At whole, these results suggest that a dorsolateral PFC hypo-functioning in older adults underlie impairment in the execution of the refreshing process. They suggest the study of the neural correlates of the refresh process might be relevant to better understand prefrontal dysfunctions in schizophrenia.

In two previous behavioral studies, we investigated the impact of refreshing on long term memory and the subjective states of awareness during recognition in schizophrenia (Grillon et al., 2005, 2010). Results showed that patients were disproportionately slower than controls in the refresh condition. Interestingly, and in contrast to older healthy adults, their ability to subsequently remember words could benefit from refreshing (Grillon et al., 2005). 
Thus, although patients were slower to refresh, requiring patients to refresh helped them to improve long term memory performance. These results suggest that patients with schizophrenia can efficiently execute refreshing (and benefit from its use) and are only impaired in engaging or initiating the refreshing process.

In the present study, we aim to better understand abnormal cerebral activations during working memory tasks by exploring the elementary refreshing task. We have used the same refreshing task used in our previous studies (Grillon et al., 2005, 2010) adapted to an fMRI experiment. Consistent with the PFC inefficiency assumption, we hypothesize refreshing is associated with an increased PFC activation in patients with schizophrenia. Second, we aim to better understand cerebral bases of the initiation impairment in refreshing of patients with schizophrenia. We hypothesize refreshing is associated with lower functional connectivity between anterior and posterior parts of the PFC (Raye et al., 2007; Koshino et al., 2011) and/or between the PFC and the inferior parietal lobule (Miller et al., 2008).

\section{Methods}

\subsection{Subjects}

The study included 15 right-handed outpatients ( 9 men, 6 women). Their mean age was 29 years (S.D.=9.2), and their mean educational level was 11.9 years (S.D.=1.8). All patients fulfilled the DSM-IV criteria for chronic schizophrenia as determined by consensus between the current treating psychiatrist and two senior psychiatrists belonging to the research team. Psychiatric symptoms were assessed by means of the Brief Psychiatric Rating Scale (mean score $=52$, S.D.=10.3; Overall and Gorham, 1962) and the Positive and Negative Syndrome Scale (mean positive=17, S.D.=7; mean negative=26, S.D.=8; mean general=44, S.D.=11; mean score=88, S.D.=19; Kay et al., 1987). Their mean age of onset was 22.6 (S.D.=7.3), their mean duration of illness was 5.4 years $($ S.D. $=4.7)$, their mean total duration of hospitalization was 6 months (S.D.=8.6) and their mean number of hospitalizations was 1.5 (S.D.=2.2). Patients with a history of traumatic brain injury, epilepsy, alcohol or substance abuse, and other diagnosed neurological conditions were excluded from the study. Patients were clinically stable on maintenance antipsychotic medication exclusively (14 on atypical antipsychotics and one on both conventional and atypical antipsychotics; mean chlorpromazine equivalent dose $=318 \mathrm{mg} /$ day, S.D.=162; Gardner et al., 2010).

The comparison group comprised 15 healthy subjects ( 9 men, 6 women). Healthy controls were screened for medical, neurological and psychiatric history by comprehensive assessment procedures. Their mean age was 29.7 years (S.D.=7.2) and their mean educational level was 12.7 years $($ S.D. $=1.6)$. The groups did not differ significantly in age $(F(1,28)=0.06, P=0.81)$ or education $(F(1,28)=1.64, P=0.21)$. The mean intelligence quotient (IQ) as assessed with the Wechsler Adult Intelligence Scale III (Wechsler, 1997) did not differ significantly between groups (mean=87.8, S.D.=16.4 in patients; mean=100.2, S.D. $=18.8$ in controls; $F(1,25)=3.24$, $P=0.08)$.

The protocol was approved by the ethical committee of the Pitié-Salpêtrière hospital. All participants provided informed written consent after the procedure had been fully explained. 


\subsection{Experimental design}

\subsubsection{Procedure}

\subsubsection{Phase 1: Refresh task}

\begin{tabular}{|l|l|l|l|l|l|l|l|l|l|l|}
\hline Word & Delay & $\begin{array}{l}\text { New word } \\
\text { Same word } \\
\text { or } \bullet\end{array}$ & & $\rightarrow$ & & $\leftarrow$ & & $\begin{array}{l}\text { Blank } \\
\text { screen }\end{array}$ \\
\hline 1450 & 550 & 1450 & 550 & 1400 & 600 & 1400 & 600 & 1400 & 600 & 2000 \\
\hline
\end{tabular}

Fig. 1: Sequence and timing (in milliseconds) of the events in a trial.

The presentation of stimuli was controlled by the E-Prime software package (Psychology Software Tools, Pittsburgh, USA). Subjects lying in the scanner viewed the computer screen through MR-compatible video goggles (VisuaStim XGA, Resonance Technology Inc., Los Angeles, USA). The sequence of each trial was strictly the same as in the Raye et al. (2002) study (see Fig. 1). Each trial was $12 \mathrm{~s}$ long. Participants silently read a word presented for $1450 \mathrm{~ms}$, followed $550 \mathrm{~ms}$ later by a new word (read trial), a repetition of the word (repeat trial), or a dot that signaled participants to think of the word that preceded the dot (refresh trial). In each case, the second stimulus was presented for $1450 \mathrm{~ms}$. The second stimulus was followed $550 \mathrm{~ms}$ later by a series of three arrows (each presented for $1400 \mathrm{~ms}$ followed by a $600 \mathrm{~ms}$ blank screen) and then a final blank screen $(2000 \mathrm{~ms})$. Whenever participants saw a word on the screen, they had to read it silently to themselves; when they saw a black dot, they had to think of the just-previous word; when they saw an arrow, they had to push a button with their left hand if the arrow pointed left and with their right hand if it pointed right. The arrows provided an $8 \mathrm{~s}$ task common to all conditions to allow time for the hemodynamic response associated with reading, repeating or refreshing words, and to decrease variability among participants due to uncontrolled mental activity between trials.

There were four runs of 30 trials each (10 per condition). In each run, read, repeat, and refresh trials were pseudo-randomly intermixed, with different orders for participants within groups (patients vs. controls) and parallel orders between groups. Across participants, each word occurred in each of the three conditions. Before the scanning session, all subjects received a practice task to check whether they had correctly understood the instructions and to familiarize them with the procedure. Neither patients nor controls made any errors (i.e. omit to say the expected word or say another word than the expected word).

Words were chosen from a pool of 200 common French two-syllable nouns, each between 4 and 10 letters in length. This word set was randomly divided into five subsets of 40 items each, which did not differ in mean word frequency or mean number of letters $(F<1)$. Each subset was presented equally often in each experimental condition and as new words in the recognition task.

\subsubsection{Phase 2: Recognition task}

About 30 min after participants exited the scanner, there was a surprise old/new recognition test consisting of 120 old words (40 from each condition) and 40 new words intermixed in a pseudorandom order. The procedure of this recognition test was exactly the same as the procedure we used in a previous study (Grillon et al., 2005). Each word on the test list appeared on the screen until the subjects pressed the button for a Yes response if they recognized the word as having occurred during the learning phase or a No response if they did not recognize the word. If the response was Yes, the subjects then pressed one of three other 
buttons labeled Remember, Know and Guess (Tulving, 1985). Then the next word appeared. If the response was No, the next word appeared immediately.

\subsubsection{Image acquisition}

Anatomical inversion recovery of T1-weighted images [3D spoiled gradient recalled (SPGR)] were acquired for each participant on a 1.5-T scanner (General Electric Health Care) using the following parameters: $\mathrm{TR} / \mathrm{TI} / \mathrm{TE}=12.4 / 450 / 2.2 \mathrm{~ms}$; flip angle $=15^{\circ}$; field of view $=240 * 240$ $\mathrm{mm}$; voxels size $=0.93 * 0.93 * 1.2 \mathrm{~mm}$; matrix $=256 * 256$. Functional scans were acquired with a single-shot echo-planar gradient-echo-pulse sequence $(\mathrm{TR}=2000 \mathrm{~ms}, \mathrm{TE}=35 \mathrm{~ms}$, flip angle $=65^{\circ}$, field of view $\left.=24 \mathrm{~cm}\right)$. The 30 axial slices $(3.8 \mathrm{~mm}$ thick) were aligned with the anterior commissure-posterior commissure line. Each run began with $12 \mathrm{sec}$ of blank screen to allow tissue to reach steady state magnetization. For each participant, 240 volumes of data, one volume every $2 \mathrm{~s}$ (six full brain scans for each trial), were collected in each condition.

\subsection{3. fMRI analyses}

Data were processed with the SPM5 software. The first six volumes of each functional session were discarded. The remaining functional images were corrected for slice timing differences using the first slice as reference (SPM5's Fourier phase shift interpolation) and realigned to the first functional image. The anatomical images were coregistered to the functional images and then spatially normalized to the MNI T1 template. The normalization parameters were then applied to the corrected functional images, which were finally smoothed with an 8-mm Gaussian kernel. The data were modeled using the canonical SPM hemodynamic response function convolved with the three experimental conditions (read, refresh, repeat). For first-level analysis, we computed single-subject voxel-wise t-statistics for the contrasts read minus refresh, repeat minus refresh, refresh minus read and refresh minus repeat using the general linear model. These single-subject contrast images were smoothed with an 8-mm Gaussian kernel (Mikl et al., 2008) and then taken to a second-level group analysis in which intersubject variability was treated as random effect (random effects analysis). Within group (one sample t-tests) and between group (two sample t-tests) analyses were carried out. Statistical parametric maps were tested for significance using cluster-level inference (cluster-defining threshold of $p<0.001$, uncorrected; cluster probability of $p<0.05$, FWE-corrected for multiple comparisons).

To clarify group differences in the refreshing-related network, we carried out region of interest (ROI) between group analysis of activation and connectivity for the contrast refresh minus read. Using the Marsbar toolbox for SPM5 (http://marsbar.sourceforge.net), we built 14 spheres of $5 \mathrm{~mm}$ in ROIs selected among regions previously associated with refreshing (Johnson et al., 2002, 2003, 2005; Raye et al., 2007). There were four spheres in the frontal cortex, six spheres in the parietal cortex, two spheres in the temporal gyrus and two spheres in the insula (see Fig. 3). We carried out the ROI analysis of activation using the Marsbar toolbox. The mean beta values for the contrast refresh minus read of all voxels within the ROIs were extracted for each subject and these measures were used to perform the between group comparison. Data for each region were thresholded at $p<0.05$ with Bonferroni correction for the number of regions evaluated.

For connectivity analysis, we rely on the standard approach of Rissman et al. (2004), modeling task-specific functional connectivity by studying trial-to-trial fluctuations in the BOLD response. For this purpose, we computed the response (beta maps) with first-level statistical inference for read, refresh and repeat experimental conditions using one regressor per stimulus. Using spheres of $5 \mathrm{~mm}$, we extracted in the 14 ROIs the brain-activation response specific to each stimulus. We then performed a second-level analysis modeling pair- 
wise correlations between the responses in each of the ROIs, i.e. the model parameters of the first-level. Finally, we modeled the dependence on the different conditions and groups for each pair-wise correlation using a random effects analysis. In the statistical framework of general linear models, we performed a t-test between groups for the contrast refresh minus read using corresponding contrast vectors and modeling common subject effect between different stimuli in the same session with a confound regressor. The significance of this t-test was assessed using non-parametric permutation tests with a t-max correction for multiple comparisons (Nichols and Holmes, 2002).

\section{Results}

\subsection{Behavioral results}

The ANOVA carried out on corrected Yes responses (correctly recognized words minus false recognitions of new words during Phase 2) showed significant Group $(F(1,27)=5.33, p=0.03)$ and Condition $(F(2,54)=13.44, p<0.001)$ effects. The main Group effect indicated that patients with schizophrenia recognized fewer words than control subjects. The main Condition effect indicated that both patients and controls recognized more refreshed and repeated words than read words (Fisher LSD following ANOVA, $d f=54, p_{s}<0.001$ ). The fact that patients with schizophrenia benefit from refreshing in the recognition memory task shows that they efficiently executed refreshing during the fMRI scanning session.

\section{2. $f M R I$ results}

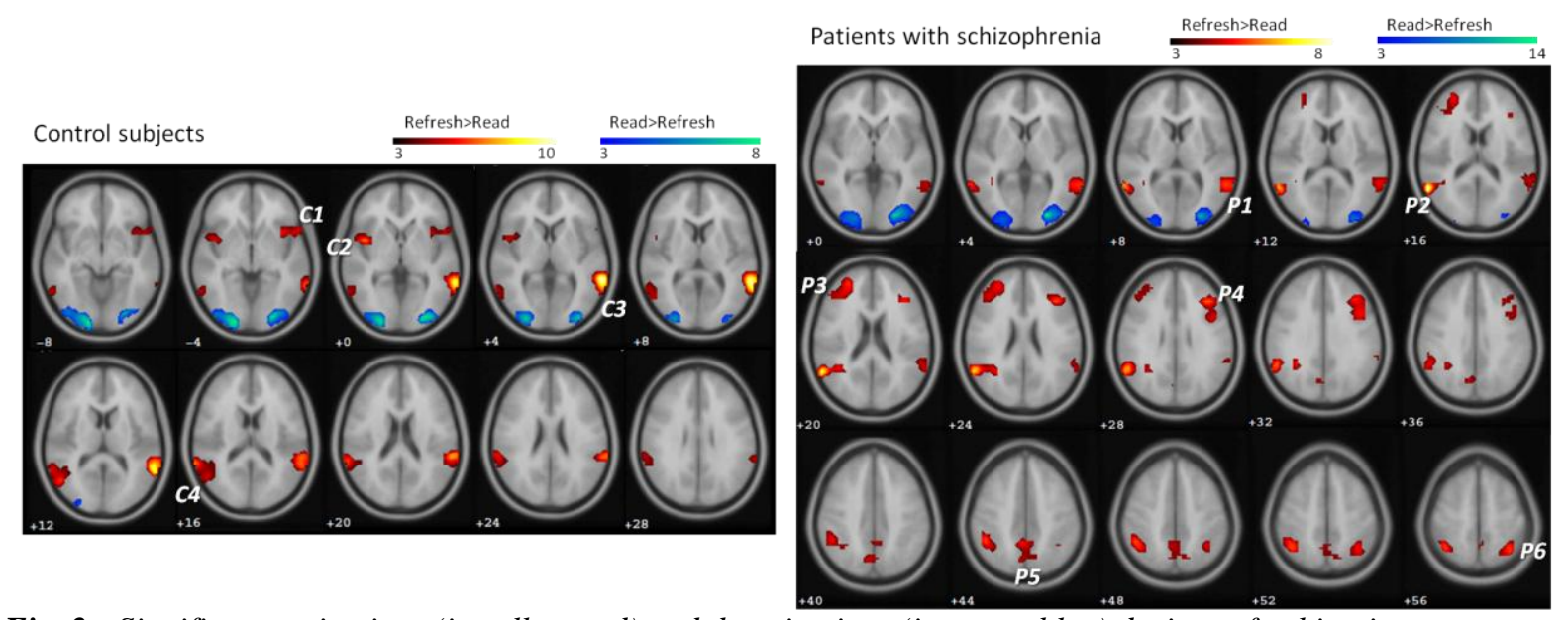

Fig. 2: Significant activations (in yellow-red) and deactivations (in green-blue) during refreshing in comparison with reading in control subjects (top) and patients with schizophrenia (bottom) $(p<0.05$ corrected at the cluster-level). C1 to C4 and P1 to P6 indicate peaks of activation in control subjects (see Table 1) and patients with schizophrenia (see Table 2), respectively.

\subsubsection{Within group analysis (see Fig. 2)}

\subsubsection{Contrast analysis in control subjects (see Table 1)}

As expected, the read and repeat conditions relative to the refresh condition activated the occipital lobe and no other cerebral regions. Greater activity for the refresh than for the read condition was observed in the bilateral insula, inferior frontal gyri, superior and middle temporal gyri and supramarginal gyri. Greater activity for the refresh than for the repeat 
condition was observed in the left superior and middle frontal gyri, the left precentral gyrus, the bilateral superior motor area, the bilateral superior and middle temporal gyri, the left inferior temporal gyrus, the bilateral supramarginal gyri, the left inferior parietal lobule, the right cuneus and the bilateral calcarine cortices.

\begin{tabular}{|c|c|c|c|c|c|c|c|}
\hline \multirow[b]{2}{*}{ Anatomical area } & \multirow[b]{2}{*}{ BA } & \multirow[b]{2}{*}{$\mathrm{k}$} & \multicolumn{3}{|c|}{$\begin{array}{l}\text { MNI coordinates for } \\
\text { peak locations }\end{array}$} & \multirow[b]{2}{*}{$\begin{array}{l}\text { Peak } T \\
\text { value }\end{array}$} & \multirow[b]{2}{*}{$\begin{array}{c}\text { p corrected cluster } \\
\text { level }\end{array}$} \\
\hline & & & $\mathrm{x}$ & $\mathrm{y}$ & $\mathrm{Z}$ & & \\
\hline \multicolumn{8}{|l|}{ Read minus Refresh } \\
\hline $\begin{array}{l}\mathrm{L} \text { middle and inferior occipital } \\
\text { gyrus }\end{array}$ & 17 & 214 & -16 & -96 & -8 & 8.00 & $<.001$ \\
\hline $\begin{array}{l}\mathrm{R} \text { middle and inferior occipital } \\
\text { gyrus }\end{array}$ & 18 & 98 & 24 & -88 & -4 & 7.56 & .002 \\
\hline $\begin{array}{l}\text { Repeat minus Refresh } \\
\text { L middle and inferior occipital } \\
\text { gyrus }\end{array}$ & 18 & 53 & -24 & -100 & 0 & 6.81 & $<.001$ \\
\hline \multicolumn{8}{|l|}{ Refresh minus Read } \\
\hline $\begin{array}{l}\mathrm{R} \text { insula, inferior frontal gyrus } \\
(C 1)\end{array}$ & 47 & 61 & 40 & 16 & -4 & 5.37 & .017 \\
\hline $\begin{array}{l}\mathrm{L} \text { insula, temporal pole superior, } \\
\text { rolandic operculum, inferior } \\
\text { frontal gyrus }(C 2)\end{array}$ & 13 & 48 & -44 & 4 & 0 & 7.06 & .039 \\
\hline $\begin{array}{l}\mathrm{R} \text { superior and middle temporal } \\
\text { gyrus, supramarginal gyrus }(C 3)\end{array}$ & 22,40 & 203 & 64 & -44 & 4 & 9.82 & $<.001$ \\
\hline $\begin{array}{l}\text { L superior and middle temporal } \\
\text { gyrus, supramarginal gyrus }(C 4)\end{array}$ & $22,39,40$ & 219 & -68 & -48 & 16 & 6.9 & $<.001$ \\
\hline \multicolumn{8}{|l|}{ Refresh minus Repeat } \\
\hline $\begin{array}{l}\text { L superior, middle and inferior } \\
\text { temporal gyrus, supramarginal } \\
\text { gyrus }\end{array}$ & 37,22 & 340 & -56 & -60 & -8 & 7.67 & $<.001$ \\
\hline $\mathrm{R}$ cuneus, $\mathrm{L}$ and $\mathrm{R}$ calcarine & 18,19 & 54 & 12 & -92 & 12 & 5.76 & .02 \\
\hline $\begin{array}{l}\text { R superior and middle temporal } \\
\text { gyrus, supramarginal gyrus }\end{array}$ & 22,40 & 256 & 64 & -48 & 12 & 6.69 & $<.001$ \\
\hline L inferior parietal lobule & 40 & 89 & -44 & -40 & 44 & 7.38 & .002 \\
\hline $\begin{array}{l}\text { L superior and middle frontal } \\
\text { gyri, precentral gyrus, L and } \mathrm{R} \\
\text { superior motor area }\end{array}$ & 6 & 212 & -8 & 4 & 60 & 8.08 & $<.001$ \\
\hline
\end{tabular}

Table 1: Areas of activation in control subjects ( $p<0.05$ corrected at the cluster-level). Peaks of activation in the refresh minus read are labeled $C 1$ to $C 4$ and depicted on Figure $2 . L=l e f t ; R=$ right; BA=Brodmann's area; $k=$ cluster size (number of voxels)

As a whole, these results are consistent with previous reports (Raye et al., 2002; Johnson et $a l ., 2005)$ and show that refreshing compared to reading or repeating activate lateral areas of the PFC, the inferior parietal lobules and temporal gyri.

\subsubsection{Contrast analysis in patients with schizophrenia (see Table 2)}

Read and repeat conditions relative to the refresh condition in patients with schizophrenia activated the occipital lobe and no other cerebral regions. This suggests that perceptual processing of the words was not significantly different between patients and control subjects. Patients with schizophrenia showed greater activity for the refresh than for the read condition in the bilateral middle and inferior frontal gyri, the bilateral middle temporal gyri, the right superior temporal gyrus, the bilateral inferior parietal lobules, supramarginal and angular gyri and precunei and the right superior parietal lobule. 
These results show refreshing in patients with schizophrenia activate lateral areas of the PFC, the inferior parietal lobules and the temporal gyri as in control subjects. However, the refreshing cerebral network was less precise and more widely distributed within the prefrontal and parietal corci in patients with schizophrenia than in control subjects.

\begin{tabular}{|c|c|c|c|c|c|c|c|}
\hline \multirow[b]{2}{*}{ Anatomical area } & \multirow[b]{2}{*}{ BA } & \multirow[b]{2}{*}{$\mathrm{k}$} & \multicolumn{3}{|c|}{$\begin{array}{l}\text { MNI coordinates for } \\
\text { peak locations }\end{array}$} & \multirow[b]{2}{*}{$\begin{array}{l}\text { Peak } T \\
\text { value }\end{array}$} & \multirow[b]{2}{*}{$\begin{array}{c}\text { p corrected cluster } \\
\text { level }\end{array}$} \\
\hline & & & $\mathrm{x}$ & $\mathrm{y}$ & $\mathrm{z}$ & & \\
\hline \multicolumn{8}{|l|}{ Read minus Refresh } \\
\hline $\begin{array}{l}\mathrm{R} \text { middle and inferior occipital } \\
\text { gyrus }\end{array}$ & 18 & 163 & 24 & -88 & 4 & 14.15 & $<.001$ \\
\hline $\begin{array}{l}\text { L middle and inferior occipital } \\
\text { gyrus }\end{array}$ & 18 & 239 & -24 & -88 & -8 & 10.47 & $<.001$ \\
\hline \multicolumn{8}{|l|}{ Repeat minus Refresh } \\
\hline $\begin{array}{l}\mathrm{R} \text { middle and inferior occipital } \\
\text { gyrus }\end{array}$ & 18 & 122 & 24 & -88 & -4 & 10.24 & $<.001$ \\
\hline $\begin{array}{l}\text { L middle and inferior occipital } \\
\text { gyrus }\end{array}$ & 17 & 213 & -20 & -92 & -8 & 10.14 & $<.001$ \\
\hline \multicolumn{8}{|l|}{ Refresh minus Read } \\
\hline $\begin{array}{l}\mathrm{R} \text { middle and superior temporal } \\
\text { gyri, supramarginal gyrus }(P l)\end{array}$ & 22,40 & 128 & 56 & -52 & 8 & 5.33 & .002 \\
\hline $\begin{array}{l}\text { L inferior parietal lobule, middle } \\
\text { temporal gyrus, angular and } \\
\text { supramarginal gyri }(P 2)\end{array}$ & $40,22,21$ & 326 & -60 & -56 & 16 & 7.82 & $<.001$ \\
\hline $\begin{array}{l}\mathrm{L} \text { middle and inferior frontal } \\
\text { gyrus }(P 3)\end{array}$ & 10,46 & 119 & -28 & 48 & 20 & 5.12 & .002 \\
\hline $\begin{array}{l}\mathrm{R} \text { middle and inferior frontal } \\
\text { gyrus }(P 4)\end{array}$ & 9 & 124 & 36 & 28 & 28 & 5.57 & $<.001$ \\
\hline $\mathrm{L}$ and $\mathrm{R}$ precunei $(P 5)$ & 7 & 112 & -4 & -48 & 44 & 4.89 & .003 \\
\hline $\begin{array}{l}\mathrm{R} \text { superior and inferior parietal } \\
\text { lobules, angular gyrus }(P 6)\end{array}$ & 7 & 59 & 36 & -56 & 56 & 5.32 & .038 \\
\hline \multicolumn{8}{|l|}{ Refresh minus Repeat } \\
\hline $\begin{array}{l}\mathrm{L} \text { precuneus, inferior and } \\
\text { superior parietal lobules, cuneus }\end{array}$ & 7,40 & 91 & -8 & -68 & 36 & 5.03 & .003 \\
\hline
\end{tabular}

Table 2: Areas of activation in patients with schizophrenia ( $p<0.05$ corrected at the cluster-level). Peaks of activation in the refresh minus read are labeled P1 to P6 and depicted on Figure 2. L = left; $R=$ right;

$B A=$ Brodmann's area; $k=$ cluster size (number of voxels)

\subsection{2. fMRI between-group analysis (see Fig. 3)}

\subsubsection{Contrast analysis}

Whole brain analysis did not reveal significant difference between groups. When the analysis was restricted to the 14 refresh-related ROIs, we observed that patients with schizophrenia showed greater activations than controls for the refresh relative to the read condition in the left inferior/middle frontal gyrus (ROI value of BA46 extracted at [ -362816$], T$ value $=3.55$, $p$ corrected for multiple comparison=0.009). No other result was significant $\left(T_{s}<2.68\right.$, $\left.p_{s}>0.08\right)$.

\subsubsection{Connectivity analysis}

Patients with schizophrenia showed reduced connectivity between the left inferior/middle frontal gyrus (BA46) and the left superior frontal gyrus (BA10) and between the left middle 
frontal gyrus (BA9) and the left inferior parietal lobule (BA40) in comparison with controls. However, they showed an increased connectivity between the left inferior parietal lobule and the supramarginal gyrus in comparison with controls.
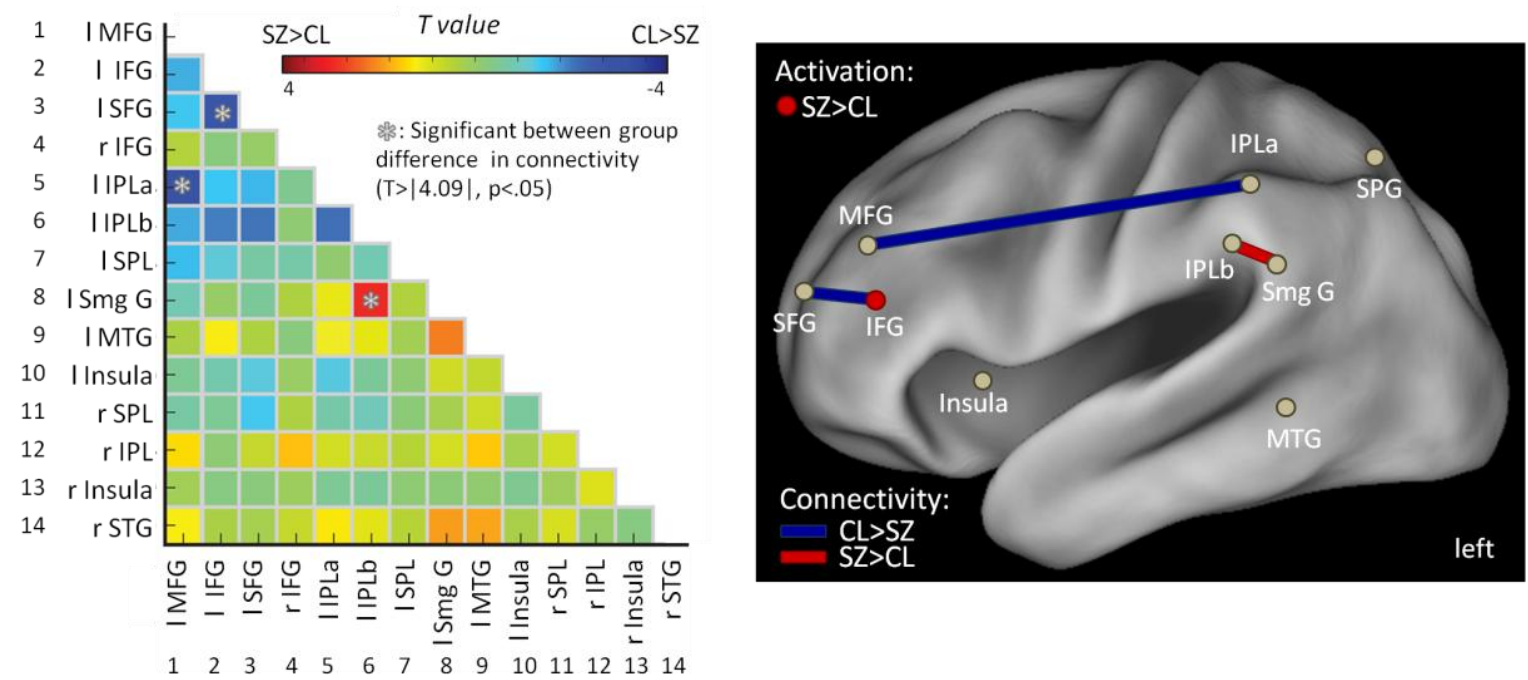

Fig. 3: Between group analyses of activations and connectivity within the refresh-related network during refreshing in comparison with reading. (A) Correlation matrix between the 14 selected regions of interest in patients with schizophrenia (SZ) compared to control subjects $(C L)$. (B) Connectivity and activation differences between patients and control subjects, a blue line indicates a decrease of connectivity in patients with schizophrenia relative to control subjects, a red line indicates an increase of connectivity in patients with schizophrenia relative to control subjects and a red point indicates an increase of activation in patients with schizophrenia relative to control subjects. Coordinates of the 14 selected ROIs: 1 (-40 $36 \quad 30), 2$ (-36 28 16), 3 (-34 50 22), 4 (46 10 -3), 5 (-46 -43 43), 6 (-46 -37 31), 7 (-28 -68 48), 8 (-62 -51 25), $9\left(\begin{array}{llll}-53 & -51 & -5\end{array}\right), 10$ (-36 7 6), 11 (36 -52 52), 12 (58 -28 23), 13 (45 16 -1), 14 (64 -36 20). l=left; r=right; MFG=Middle Frontal Gyrus; IFG=Inferior Frontal Gyrus; SFG=Superior Frontal Gyrus; IPL=Inferior Parietal Lobule; $S P L=$ Superior Parietal Lobule; Smg G=Supramarginal gyrus; MTG=Middle Temporal Gyrus; $S T G=$ Superior Temporal Gyrus.

\section{Discussion}

Consistent with previous findings (Raye et al., 2002; Johnson et al., 2005), the brain network underlying refreshing in control subjects involved the lateral PFC, the inferior parietal lobules/supramarginal gyri and the temporal gyri. Although the refresh-related brain network in patients with schizophrenia was less well circumscribed than in control subjects, it also involved frontal, parietal and temporal cerebral areas.

The between group contrast analysis restricted to the refresh-related network shows that refreshing (relative to reading) was associated with a hyper-activation in the left inferior/middle frontal gyrus (BA46) in patients with schizophrenia compared to control subjects. This result is consistent with the PFC inefficiency hypothesis that predicts PFC hyper-activations during low-load working memory tasks such as the refreshing task (Callicott et al., 2003; Manoach, 2003). According to this PFC inefficiency hypothesis, hyperfrontality would reflect the fact that patients with schizophrenia devote more blood oxygen level dependent response than control participants to achieve a performance equivalent to that of control participants when the task load does not exceed their cognitive capacities. 
Evidence from behavioral studies suggested that the underlying mechanisms of the impairment of refreshing were different between patients with schizophrenia and normal aged adults (Johnson et al., 2002; Johnson et al., 2004; Grillon et al., 2005, 2010). For instance, long term memory benefited from refreshing in patients with schizophrenia but not in older adults. The present results provide evidence that the neural correlates of the refreshing process are different between the two populations: refreshing is associated with an hypo-frontality in older adults (Johnson et al., 2004) whereas it is associated with an hyper-frontality in patients with schizophrenia.

It has been suggested that patients were impaired in engaging or initiating the refreshing process but were able to efficiently execute it (Grillon et al., 2005, 2010) whereas normal aged adults were impaired in executing it (Johnson et al., 2002). Using repetitive transcranial magnetic stimulation, Miller et al. (2008) showed a selective slowing of response times to refresh words following the left middle frontal gyrus stimulation. These results are in favor of a role of the PFC as the source of the top-down signals to more posterior areas necessary for refreshing. Interestingly, a critical result of our study is a reduced connectivity between the left dorsolateral PFC and the left inferior parietal lobule in patients with schizophrenia relative to control participants when the refresh condition was compared with the read condition. Altogether, these findings suggest that the slowing of refresh latencies in schizophrenia could be due in part to a fronto-parietal connectivity disruption. This disruption could prevent the left dorsolateral PFC from efficiently controlling activity in other refreshrelated brain regions. However, reduced fronto-parietal connectivity in schizophrenia has also been reported during lexical decision, retrieval in long term memory (Foucher et al., 2005), choice reaction time (Woodward et al., 2009), N-Back (Tan et al., 2006), Sternberg (Kim et al., 2009), non-articulatory maintenance of phonological information (Henseler et al., 2010), AX Continuous Performance (Fornito et al., 2011) and verbal working memory (Deserno et al., 2012) tasks. Even if it should be noted that the refreshing of task-relevant information is important for all these tasks, it remains possible that the fronto-parietal abnormalities reported in schizophrenia are related to more general impairments in cognitive control or in the allocation of attention.

Patients with schizophrenia also showed a decrease in functional connectivity between the left frontopolar area (BA10) and a more posterior area of the prefrontal cortex, the anterior portion of the left inferior/middle prefrontal gyrus (BA46). This is consistent with previous reports of altered connectivity within the prefrontal cortex during cognitive tasks in schizophrenia (Henseler et al., 2010; Barbalat et al., 2011). In addition, studies in healthy participants suggested that the anterior prefrontal cortex is involved in initiating the refresh process (Raye et al., 2007, experiment 1; Johnson et al., 2005; see also Koshino et al., 2011), while more posterior regions are involved in executing the refresh process (Raye et al., 2007; Johnson et al., 2005). Although the PFC network involved in task initiation is not fully understood (but see Kouneiher et al., 2009), these findings suggest that the impairment of initiation in the present study could result from a defective connectivity between anterior and posterior parts of the PFC in schizophrenia.

Taken together the present findings show refreshing is associated with prefrontal hyperactivation and both inter-regional and intra-regional hypoconnectivity. Although prefrontal dysfunctions have been related to inefficient execution of cognitive processing in schizophrenia, the present findings show prefrontal dysfunctions might be related to an impaired ability to initiate (but not to execute) a single elementary process (see also Hirst and Volpe, 1988; Kirchhoff, 2009). Consistent with these results, when patients are forced to use an efficiently executed but not spontaneously engaged strategy (e.g. semantic clustering), patients with schizophrenia still show prefrontal dysfunctions (Bonner-Jackson et al., 2005). 
Moreover, studies using a component process approach of complex cognitive tasks identify island of efficiently initiated PFC-taxing processes - such as orienting spatial attention (Gold et al., 2009), reactive inhibition (Zandbelt et al., 2011) and articulatory rehearsal (Henseler $e t$ al., 2010; Bacon et al., 2007) - whose the use is not associated with prefrontal dysfunctions. Taken together, these results suggest that prefrontal dysfunctions in schizophrenia might not be truly a primary and specific deficit but occur during the use of specific processes impaired in their initiation. In other words, prefrontal dysfunctions might be secondary consequence of a diffuse deficit that affects the initiation of specific processes. In this regard, a recent study suggests that the impaired ability to initiate refreshing can be linked with the increase in the amount and/or distance of spreading activation within semantic memory in schizophrenia (Higgins and Johnson, 2012): the increased automatic spreading of activation of representations semantically related to the target would interfere with the target refreshing. Future studies are thus needed to better characterize the PFC-taxing processes whose use is associated with PFC dysfunctions in comparison with PFC-taxing processes whose use is not associated with PFC dysfunctions in schizophrenia.

Limitations of the current study must be acknowledged. First the present study uses a relatively small sample size. Thus, the absence of between group differences with the whole brain analyses may be a result of low statistical power. However, as the cerebral bases of the refreshing process are well known in control subjects, we carried out an ROI analysis. This allowed us to improve the statistical power of our between group analysis. Second, it could be asked whether medication or specific symptoms are related to the cerebral bases of the refreshing impairment in schizophrenia. Although this cannot be fully rejected, the fact that we did not show correlations of the refreshing behavioral measures (e.g. response times) with symptoms or drug dose in our previous studies (Grillon et al., 2005) argues against these possibilities. Last, it is possible that our findings might prove to be specific to refreshing visual words. It will thus remain for future studies to determine if this can be generalized to other kinds of information such as images and auditory words.

To conclude, the use of a carefully controlled elementary working memory task, i.e. the refreshing task, allowed us to highlight brain circuitry group differences which cannot be due to the use of different strategies or processes between groups. The defective ability to initiate refreshing in schizophrenia was associated with an increased activation of the left inferior/middle frontal gyrus (BA46) and an impaired functional connectivity within the refreshing network.

\section{Acknowledgments}

The authors gratefully thank Christophe Pallier, for his help with fMRI analyses, Marcia Johnson and Carol Raye for their relevant comments on data, Virginie Van Wassenhove, Marco Sperdutti and the two anonymous reviewers for their helpful comments and suggestions for improving the manuscript. This research was supported by the Sainte Anne Hospital délégation à la recherche. MLG reports having received a research funding from the FRM (Fondation pour la Recherche Médicale) and the award "for women in science" from the foundation l'Oréal France-Unesco-Académie des sciences.

\section{Declaration of Interest}

None. 


\section{References}

1. Andreasen, N.C., Nopoulos, P., O'Leary, D.S., Miller, D.D., Wassink, T., Flaum, M., 1999. Defining the phenotype of schizophrenia: cognitive dysmetria and its neural mechanisms. Biological Psychiatry 46, 908-920.

2. Anticevic, A., Repovs, G., Barch, D.M., 2011. Working Memory Encoding and Maintenance Deficits in Schizophrenia: Neural Evidence for Activation and Deactivation Abnormalities. Schizophrenia Bulletin.

3. Barbalat, G., Chambon, V., Domenech, P.J., Ody, C., Koechlin, E., Franck, N., Farrer, C., 2011. Impaired hierarchical control within the lateral prefrontal cortex in schizophrenia. Biological Psychiatry 70, 73-80.

4. Callicott, J.H., Egan, M.F., Mattay, V.S., Bertolino, A., Bone, A.D., Verchinksi, B., Weinberger, D.R., 2003. Abnormal fMRI response of the dorsolateral prefrontal cortex in cognitively intact siblings of patients with schizophrenia. American Journal of Psychiatry 160, 709-719.

5. Camos, V., Mora, G., Oberauer, K., 2011. Adaptive choice between articulatory rehearsal and attentional refreshing in verbal working memory. Memory \& Cognition 39, 231-244.

6. Chun, M.M., Johnson, M.K., 2011. Memory: enduring traces of perceptual and reflective attention. Neuron $72,520-535$.

7. Conway, A.R.A., Jarrold, C., Kane, M.J., Miyake, A., Towse, J., 2007. Variation in Working Memory. Oxford University Press, U.K.

8. Edwards, B.G., Barch, D.M., Braver, T.S., 2010. Improving prefrontal cortex function in schizophrenia through focused training of cognitive control. Frontiers in Human Neuroscience 4, 32.

9. Eisenberg, D.P., Berman, K.F., 2010. Executive function, neural circuitry, and genetic mechanisms in schizophrenia. Neuropsychopharmacology 35, 258-277.

10. Fornito, A., Yoon, J., Zalesky, A., Bullmore, E.T., Carter, C.S., 2011. General and specific functional connectivity disturbances in first-episode schizophrenia during cognitive control performance. Biological Psychiatry 70, 64-72.

11. Foucher, J.R., Vidailhet, P., Chanraud, S., Gounot, D., Grucker, D., Pins, D., Damsa, C., Danion, J.M., 2005. Functional integration in schizophrenia: too little or too much? Preliminary results on fMRI data. Neuroimage 26, 374-388.

12. Friston, K., 2005. Disconnection and cognitive dysmetria in schizophrenia. American Journal of Psychiatry 162, 429-432.

13. Grillon, M.L., Johnson, M.K., Danion, J.M., Rizzo, L., Verdet, C., Huron, C., 2005. Assessing a minimal executive operation in schizophrenia. Psychiatry Research 137, 37-48.

14. Grillon, M.L., Johnson, M.K., Krebs, M.O., Huron, C., 2008. Comparing effects of perceptual and reflective repetition on subjective experience during later recognition memory. Consciousness \& Cognition 17, 753-764.

15. Grillon, M.L., Krebs, M.O., Gourevitch, R., Giersch, A., Huron, C., 2010. Episodic memory and impairment of an early encoding process in schizophrenia. Neuropsychology 24, 101-108.

16. Haut, K.M., Lim, K.O., MacDonald, A., 3rd, 2010. Prefrontal cortical changes following cognitive training in patients with chronic schizophrenia: effects of practice, generalization, and specificity. Neuropsychopharmacology 35, 1850-1859.

17. Henseler, I., Falkai, P., Gruber, O., 2009. A systematic fMRI investigation of the brain systems subserving different working memory components in schizophrenia. European Journal of Neuroscience 30, 693-702. 
18. Henseler, I., Kruger, S., Dechent, P., Gruber, O., 2011. A gateway system in rostral PFC? Evidence from biasing attention to perceptual information and internal representations. Neuroimage 56, 1666-1676.

19. John, J.P., Halahalli, H.N., Vasudev, M.K., Jayakumar, P.N., Jain, S., 2011. Regional brain activation/deactivation during word generation in schizophrenia: fMRI study. British Journal of Psychiatry 198, 213-222.

20. Johnson, M.K., Hirst, W., 1993. MEM: Memory subsystems as processes. In Collins, A.F., Gathercole, S.E., Conway, M.A., Morris, P.E. (Eds.), Theories of Memory, Erlbaum, East Sussex, England, pp. 241-286.

21. Johnson, M.K., Mitchell, K.J., Raye, C.L., Greene, E.J., 2004. An age-related deficit in prefrontal cortical function associated with refreshing information. Psychological Science 15, 127-132.

22. Johnson, M.K., Raye, C.L., Mitchell, K.J., Greene, E.J., Anderson, A.W., 2003. FMRI evidence for an organization of prefrontal cortex by both type of process and type of information. Cerebral Cortex 13, 265-273.

23. Johnson, M.K., Raye, C.L., Mitchell, K.J., Greene, E.J., Cunningham, W.A., Sanislow, C.A., 2005. Using fMRI to investigate a component process of reflection: prefrontal correlates of refreshing a just-activated representation. Cognitive, Affective \& Behavioral Neuroscience 5, 339-361.

24. Johnson, M.K., Reeder, J.A., Raye, C.L., Mitchell, K.J., 2002. Second thoughts versus second looks: an age-related deficit in reflectively refreshing just-activated information. Psychological Science 13, 64-67.

25. Kay, S.R., Fiszbein, A., Opler, L.A., 1987. The Positive and Negative Syndrome Scale (PANSS) for schizophrenia. Schizophrenia Bulletin 13, 261-276.

26. Keeser, D., Meindl, T., Bor, J., Palm, U., Pogarell, O., Mulert, C., Brunelin, J., Moller, H.J., Reiser, M., et al., 2011. Prefrontal transcranial direct current stimulation changes connectivity of resting-state networks during fMRI. The Journal of Neuroscience 31, 15284-15293.

27. Kim, D.I., Manoach, D.S., Mathalon, D.H., Turner, J.A., Mannell, M., Brown, G.G., Ford, J.M., Gollub, R.L., White, T., et al., 2009. Dysregulation of working memory and default-mode networks in schizophrenia using independent component analysis, an fBIRN and MCIC study. Human Brain Mapping 30, 3795-3811.

28. Kim, M.A., Tura, E., Potkin, S.G., Fallon, J.H., Manoach, D.S., Calhoun, V.D., Turner, J.A., 2010. Working memory circuitry in schizophrenia shows widespread cortical inefficiency and compensation. Schizophrenia Research 117, 42-51.

29. Koshino, H., Minamoto, T., Ikeda, T., Osaka, M., Otsuka, Y., Osaka, N., 2011. Anterior medial prefrontal cortex exhibits activation during task preparation but deactivation during task execution. PLoS One 6, e22909.

30. Kouneiher, F., Charron, S., Koechlin, E., 2009. Motivation and cognitive control in the human prefrontal cortex. Nature Neuroscience 12, 939-945.

31. Libby, L.A., Ragland, J.D., 2011. fMRI as a Measure of Cognition Related Brain Circuitry in Schizophrenia. Current Topics in Behavioral Neurosciences.

32. Manoach, D.S., 2003. Prefrontal cortex dysfunction during working memory performance in schizophrenia: reconciling discrepant findings. Schizophrenia Research 60, 285-298.

33. Miller, B.T., Verstynen, T., Johnson, M.K., D'Esposito, M., 2008. Prefrontal and parietal contributions to refreshing: an rTMS study. Neuroimage 39, 436-440.

34. Minzenberg, M.J., Laird, A.R., Thelen, S., Carter, C.S., Glahn, D.C., 2009. Metaanalysis of 41 functional neuroimaging studies of executive function in schizophrenia. Archives of General Psychiatry 66, 811-822. 
35. Overall, J.E., Gorham, D.R., 1962. The Brief Psychiatric Rating Scale. Psychological Reports 10, 799-812.

36. Pettersson-Yeo, W., Allen, P., Benetti, S., McGuire, P., Mechelli, A., 2011. Dysconnectivity in schizophrenia: where are we now? Neuroscience \& Biobehavioral Reviews 35, 1110-1124.

37. Potkin, S.G., Turner, J.A., Brown, G.G., McCarthy, G., Greve, D.N., Glover, G.H., Manoach, D.S., Belger, A., Diaz, M., et al., 2009. Working memory and DLPFC inefficiency in schizophrenia: the FBIRN study. Schizophrenia Bulletin 35, 19-31.

38. Quintana, J., Wong, T., Ortiz-Portillo, E., Kovalik, E., Davidson, T., Marder, S.R., Mazziotta, J.C., 2003. Prefrontal-posterior parietal networks in schizophrenia: primary dysfunctions and secondary compensations. Biological Psychiatry 53, 12-24.

39. Raye, C.L., Johnson, M.K., Mitchell, K.J., Greene, E.J., Johnson, M.R., 2007. Refreshing: a minimal executive function. Cortex 43, 135-145.

40. Raye, C.L., Johnson, M.K., Mitchell, K.J., Reeder, J.A., Greene, E.J., 2002. Neuroimaging a single thought: dorsolateral PFC activity associated with refreshing just-activated information. Neuroimage 15, 447-453.

41. Raye, C.L., Mitchell, K.J., Reeder, J.A., Greene, E.J., Johnson, M.K., 2008. Refreshing one of several active representations: behavioral and functional magnetic resonance imaging differences between young and older adults. Journal of Cognitive Neuroscience 20, 852-862.

42. Salgado-Pineda, P., Fakra, E., Delaveau, P., McKenna, P.J., Pomarol-Clotet, E., Blin, O., 2011. Correlated structural and functional brain abnormalities in the default mode network in schizophrenia patients. Schizophrenia Research 125, 101-109.

43. Schmitt, A., Hasan, A., Gruber, O., Falkai, P., 2011. Schizophrenia as a disorder of disconnectivity. European Archives of Psychiatry Clinical Neuroscience 261 Suppl 2, S150-154.

44. Sperling, G. 1960. The information available in brief visual presentations. Psychological Monographs 74, 1-29.

45. Tan, H.Y., Choo, W.C., Fones, C.S., Chee, M.W., 2005. fMRI study of maintenance and manipulation processes within working memory in first-episode schizophrenia. American Journal of Psychiatry 162, 1849-1858.

46. Tan, H.Y., Sust, S., Buckholtz, J.W., Mattay, V.S., Meyer-Lindenberg, A., Egan, M.F., Weinberger, D.R., Callicott, J.H., 2006. Dysfunctional prefrontal regional specialization and compensation in schizophrenia. American Journal of Psychiatry 163, 1969-1977.

47. Wechsler, D., 1997. WAIS III : Echelle d'intelligence pour adultes, 3d ed. ECPA: Paris, France.

48. Winterer, G., Weinberger, D.R., 2004. Genes, dopamine and cortical signal-to-noise ratio in schizophrenia. Trends in Neuroscience 27, 683-690.

49. Woodward, N.D., Rogers, B., Heckers, S., 2011. Functional resting-state networks are differentially affected in schizophrenia. Schizophrenia Research 130, 86-93.

50. Woodward, N.D., Waldie, B., Rogers, B., Tibbo, P., Seres, P., Purdon, S.E., 2009. Abnormal prefrontal cortical activity and connectivity during response selection in first episode psychosis, chronic schizophrenia, and unaffected siblings of individuals with schizophrenia. Schizophrenia Research 109, 182-190.

51. Zandbelt, B.B., van Buuren, M., Kahn, R.S., Vink, M., 2011. Reduced proactive inhibition in schizophrenia is related to corticostriatal dysfunction and poor working memory. Biological Psychiatry 70, 1151-1158. 